\title{
ON CYCLIC SUBGROUPS OF FINITE GROUPS
}

\author{
by U. DEMPWOLFF and S. K. WONG
}

(Received 13th December 1979)

In [3] Laffey has shown that if $Z$ is a cyclic subgroup of a finite subgroup $G$, then either a nontrivial subgroup of $Z$ is normal in the Fitting subgroup $F(G)$ or there exists a $g$ in $G$ such that $Z^{\mathrm{g}} \cap Z=1$. In this note we offer a simple proof of the following generalisation of that result:

Theorem. Let $G$ be a finite group and $X$ and $Y$ cyclic subgroups of $G$. Then there exists $a \mathrm{~g}$ in $G$ such that $X^{\mathrm{g}} \cap Y \unlhd F(G)$.

Remark. Note that $X$ and $Y$ need not to be conjugate subgroups of $G$. Our notation is standard (see [2]). A subgroup $H$ is said to be a component of $G$ if $[H, H]=H, H$ is subnormal in $G$ and $H / Z(H)$ is a simple group. The product of all components of $G$ is called $E(G)$. Let $F^{*}(G)=F(G) \cdot E(G)$. Then $C_{G}\left(F^{*}(G)\right) \subseteq F^{*}(G)$ (see page 165 of [1]),

Proof of Theorem. We choose $G$ to be a minimal counterexample and $X, Y$ chosen so that $\max (|X|,|Y|)$ is of minimal order. Consequently,

(I) We may assume $|X|=|Y|$ and if $X_{p}$ is a $S_{p}$-subgroup of $X$ then there exists a $g$ in $G$ such that $X_{p}^{\mathrm{g}} \in \operatorname{Syl}_{p}(Y)$.

(II) The group $X$ is of square-free order.

Suppose $p^{2}$ divides $|X|$. Then by induction, we may assume (by replacing $Y$ by a suitable conjugate) that $L=X \cap N=Y \cap N \neq 1$, where $N=\Omega_{1}\left(Z\left(O_{p}(G)\right)\right)$. First assume $L \unlhd G$. Since $C_{G}(L) \unlhd G$ and $X, Y$ are contained in $C_{G}(L)$, we have by induction that $G=C_{G}(L)$.

But by induction on $G / L$, we have a contradiction. So $L \not g G$. Let $g \in G$ such that $X^{\mathrm{g}} \cap Y$ is a $p^{\prime}$-group. Set $X_{O}^{\mathrm{g}}=O_{p^{\prime}}\left(X^{\mathrm{g}}\right)$ and $T=C_{G}(N) X_{O}^{\mathrm{g}}$. Since $N \subseteq F(T)$ and $\left(|N|,\left|X_{O}^{g}\right|\right)=1$, we have $F(T) \subseteq C_{G}(N)$. Hence $F(T)=F\left(C_{G}(N)\right)=F(G)$. By induction, there exists a $h \in C_{G}(N)$ such that $X_{O}^{\mathrm{gh}} \cap(Y \cap T) \unlhd F(T)=F(G)$. Since $L^{h}=L$, we have $X^{\mathrm{gh}} \cap Y=X_{O}^{\mathrm{gh}} \cap Y=X_{O}^{\mathrm{gh}} \cap(Y \cap T) \unlhd F(G)$, and we have a contradiction. This proves that $X$ is of square-free order.

(III) $G=F^{*}(G) X=F^{*}(G) Y$.

Let $G_{O}=F^{*}(G) X$. Since $C_{G}\left(F^{*}(G)\right) \subseteq F^{*}(G)$ and $F\left(G_{O}\right) \supseteq F(G)$, we have $Z\left(F\left(G_{O}\right)\right) \subseteq Z(F(G))$. If $G_{O} \subset G$, then by induction there exists a $g \in G_{O}$ with $X^{\mathrm{g}} \cap\left(Y \cap G_{O}\right) \unlhd F\left(G_{O}\right)$. i.e. $X^{\mathrm{g}} \cap Y \cap G_{O} \subseteq Z\left(F\left(G_{O}\right)\right) \subseteq Z(F(G))$. Since $X^{\mathrm{g}} \cap Y=$ $X^{8} \cap Y \cap G_{O}$ we are done.

(IV) If $1 \neq N \subseteq \Omega_{1}\left(Z\left(O_{p}(G)\right)\right)$ with $N \unlhd G$, then $F(G / N) \supset F(G) / N$. Suppose 
$F(G / N)=F(G) / N$. Then by induction we may assume $L=O_{p}(X \cap Y) N \unlhd F(G)$ but $O_{p}(X \cap Y) \downarrow F(G)$. Since $G=F^{*}(G) X$, we have $L \unlhd G$. Let $g \in G-N_{G}\left(O_{p}(X \cap Y)\right)$ and $T=C_{G}(L) X^{g}$. Since $O_{\mathrm{p}^{\prime}}(F(G)) \subseteq C_{G}(L)$ we have $O_{p^{\prime}}(F(G)) \subseteq O_{p^{\prime}}(F(T))$. But since $O_{p^{\prime}}(F(T)) \subseteq C_{G}(L)$ and $F\left(C_{G}(L) \subseteq F(G)\right.$, we have $O_{p^{\prime}}(F(T))=O_{p^{\prime}}(F(G))$. Now by induction, there is a $t \in T$ such that $X^{g t} \cap(T \cap Y) \unlhd F(T)$. Since $t$ centralises $O_{p}(X \cap Y)$, we have $X^{g t} \cap(T \cap Y)$ is a $p^{\prime}$-group. Hence $X^{\mathrm{gt}} \cap Y \unlhd O_{p^{\prime}}(F(G))$, a contradiction.

(V) $O_{\mathrm{p}}(F(G))$ is elementary abelian, $F(G) \cap E(G)=1$ and $X \cap F(G)=Y \cap F(G)=1$.

Suppose $O_{p}(F(G))$ is not elementary abelian. Setting $N=\phi\left(O_{\mathrm{p}}(G)\right) \cap \Omega_{1}\left(Z\left(O_{\mathrm{p}}(G)\right)\right.$, we have $F(G / N)=F(G) / N$ contradicting (IV). Similarly, if $F(G) \cap E(G) \neq 1$, let $N=$ $\Omega_{1}\left(O_{\mathrm{p}}(F(G) \cap E(G))\right)$ and again we have a contradiction by (IV). The last assertion follows by induction.

(VI) Either $F^{*}(G)=O_{p}(G)$ or $F^{*}(G)=E(G)$ is a minimal normal subgroup of $G$.

Suppose the contrary. We have the following cases:

(i) $F(G)=1$ and $1 \neq M_{1}, M_{2} \unlhd G$ such that $E(G)=M_{1} \times M_{2}$.

(ii) $E(G)=1$ and set $M_{1}=O_{p}(G)$ and $M_{2}=O_{p^{\prime}}(F(G))$.

(iii) Set $M_{1}=F(G) \neq 1$ and $M_{2}=E(G) \neq 1$.

Now let $X_{O}=C_{X}\left(M_{2}\right)$ and $Y_{O}=C_{Y}\left(M_{2}\right)$. Note that by $(I),\left|X_{O}\right|=\left|Y_{O}\right|$. As $M_{1} X_{O} \unlhd G$, we have $F^{*}\left(M_{1} X_{O}\right)=M_{1}$, and hence there exists a $f \in M_{1}$ with $X_{O}^{f} \cap Y_{O}=1$. Set $X=X_{O} \times X_{O O}, Y=Y_{O} \times Y_{O O}$ and $T=M_{2} X_{O O}^{f}$. Pick $m \in M_{2}$ such that $X_{O O}^{f} \cap Y_{O O}^{M} \unlhd$ $F(T)$. In cases (i) and (iii), $F(T) \subseteq C_{G}\left(M_{2}\right)$ and so $X_{O O}^{f} \cap Y_{O O}^{m}=1$. In case (ii) $F(T) \supseteq$ $M_{2}$ and by $(V),\left(X_{O O}^{f} \cap Y_{O O}^{m}\right) \cap M_{2}=1$ and hence $X_{O O}^{f} \cap Y_{O O}^{m}=1$. Since $Y_{O}^{m}=Y_{O}$ and $\left|X_{O}\right|,\left|X_{O O}\right|$ are coprime numbers, we have $X^{f} \cap Y^{m}=\left(X_{O}^{f} \cap Y_{O}^{m}\right) \times\left(X_{O O}^{f} \cap Y_{O O}^{m}\right)=1$, a contradiction.

(VII) Final contradiction.

If $F^{*}(G)=O_{p}(G)$, we have by the Schur-Zassenhaus theorem [2, Thm. 2.1, p. 221] that $X^{\mathrm{R}}=Y$ for some $g \in G$. By Maschke's theorem, [2, Thm. 3.1, p. 66], let $O_{p}(G)=V_{1} V_{2} \ldots V_{r}$ where $V_{i}$ are minimal $X$-invariant subgroups. Let $1 \neq v_{i} \in V_{i}$, then $X \cap X^{v_{1}, v_{2} \cdots v_{r}}=1$, a contradiction. Hence by (VI), we have $F^{*}(G)=E(G)$ is a minimal normal subgroup of $G$. Let $X_{p} \in \operatorname{Syl}_{p}(X)$. Then by (I), there exists a $g_{p}$ in $G$ such that $X^{s_{p}} \in \operatorname{Syl}_{p}(Y)$. Since $G$ is a counterexample, for any $g$ in $G$ there exists a prime $p$ such that $p \| X^{g} \cap Y \mid$. Hence $X_{p}^{g}=X_{p}^{g_{p}}$ and $g \in N_{G}\left(X_{p}\right) g_{p}$. So we have $G=\bigcup_{p \in \pi(X)} N_{G}\left(X_{p}\right) g_{p}$. Let $N_{G}\left(X_{q}\right)=\max \left(\left|N_{G}\left(X_{p}\right)\right| \mid p \in \pi(X)\right)$. Then $\left|G: N_{G}\left(X_{q}\right)\right|<\pi(X)<r=\max (\pi(X))$. Hence $1 \neq X_{r} \subseteq \bigcap_{\mathrm{g} \in G} N_{G}\left(X_{q}\right)^{\mathrm{R}}$ and so $E(G) \subseteq N_{G}\left(X_{q}\right)$, which is impossible. This final contradiction proves the theorem.

\section{REFERENCES}

1. H. Bender, On groups with Abelian Sylow 2-subgroups, Math. Z. 117 (1970), 164-176.

2. D. Gorenstein, Finite groups (Harper and Row Publishers, New York, 1968).

3. T. J. LAFFEY, Disjoint conjugates of cyclic subgroups of finite groups, Proc. Edinburgh Math. Soc. 20 (1976-1977), 229-232.

UNIVERSITÄT KAISERSLAUTERN

FB MATHEMATIK

POSTFAD 3049

D-675 KAISERSL_AuTERN
The Ohio State University Department of Mathematics 231 W. 18th AVE.

Columbus, Ohio 43210 U.S.A. 